\title{
Specific reversal of MDR1/P-gp-dependent multidrug resistance by RNA interference in colon cancer cells
}

\author{
ZHONGSHENG XIA ${ }^{1}$, ZHAOHUA ZHU ${ }^{1}$, LIYONG ZHANG ${ }^{2}$, CRISTA ROYAL $^{3}$, \\ ZHONGMIN LIU $^{2}$, QIKUI CHEN ${ }^{1}$ and BAO-LING ADAM ${ }^{2}$ \\ ${ }^{1}$ Department of Gastroenterology, The Second Affiliated Hospital, Sun Yat-sen University, Guangzhou 510120, \\ P.R. China; ${ }^{2}$ Center for Biotechnology and Genome Medicine, ${ }^{3}$ Department of Pharmacology \\ and Toxicology, Medical College of Georgia, Augusta, GA 30912, USA
}

Received May 13, 2008; Accepted June 26, 2008

DOI: 10.3892/or_00000163

\begin{abstract}
No control cell line was available for previous RNA interference studies on reversal of multidrug resistance (MDR) in colon cancer cells. Here, human COLO 320DM, with HT-29 as the control, colon cancer cell lines were used to investigate the reversal of MDR1/P-gp-dependent MDR by siRNA (\#4123 and \#4029 MDR1 siRNAs) targeting to MDR1 mRNA. Both siRNAs inhibited expression of MDR1 and P-gp in COLO 320DM. The minimum inhibition concentrations were $5 \mathrm{nmol} / \mathrm{l}$ of \#4123 and $25 \mathrm{nmol} / \mathrm{l}$ of \#4029. \#4123 MDR1 siRNA took effect in 4, 5 and 6 days at doses of 5, 25 and $100 \mathrm{nmol} / \mathrm{l}$, respectively. Increased cytotoxicity of the antitumor drugs adriamycin and vincristine with increased intracellular adriamycin accumulation accompanied inhibition of MDR1 mRNA and P-gp expression. No such effects were found in the HT-29 control. MDR1 siRNAs specifically reversed the MDR of colon cancer cells demonstrating a possible new approach for treating MDR1/P-gpdependent multidrug resistance.
\end{abstract}

\section{Introduction}

Colon cancer is the second leading cause of cancer death in the United States (1). Most patients with colon cancer are in its advanced stage by the time of diagnosis (2), so surgery is rarely sufficient treatment. Consequently, chemotherapy is very important in the treatment of colon cancer. However, the effectiveness of chemotherapy is limited because colon cancer cells become drug resistance (3).

Classical multiple drug resistance (MDR) in cancer cells is thought to be related to the overexpression of P-glycoprotein

Correspondence to: Dr Zhongsheng Xia, Department of Gastroenterology, The Second Affiliated Hospital, Sun Yat-sen University, Guangzhou 510120, P.R. China

E-mail: xiazhsh@hotmail.com

Key words: small interfering RNA, RNA interference, MDR1, $\mathrm{P}$-glycoprotein, colon cancer, multidrug resistance
(P-gp, MDR1) (4). P-glycoprotein is a 170-kDa transmembrane protein and a member of the ATP-binding cassette transporter superfamily. P-glycoprotein functions as an energydependent efflux pump, thereby resulting in decreased concentrations of intracellular drugs and resistance to the chemotherapeutic effect of these drugs. To improve the effect of chemotherapy in the treatment of colon cancer, one of the major challenges is how to reverse MDR in colon cancer cells. In previous studies, calcium antagonists such as verapamil (5), antisense oligonucleotides (6) and hammerhead ribozymes (7) were found to modulate MDR1/P-gp-dependent MDR by down-regulating the expression of the MDR1 mRNA and P-gp. However, calcium antagonists may cause heart failure and hypotension (8), and the clinical benefit of antisense oligonucleotides and hammerhead ribozymes has not been realized. So successful reversal of drug resistance is still awaiting new therapeutic strategies or pharmaceuticals.

A novel means for specific inhibition of a target gene is the use of small interfering RNA (siRNA). These 21-23 nucleotides (nt) long, double-stranded RNA (dsRNA) molecules can direct degradation of eukaryotic mRNAs in a sequence-specific manner (9). This ubiquitous mechanism of gene regulation in plants and animals was designated as RNA interference (RNAi) (10).

In this study, we chose the COLO 320DM cell line for our colon cancer model to investigate the reversal of MDR using siRNA. COLO 320DM has been reported to express P-gp and has MDR to many chemotherapeutic agents (14). The HT-29 control cell line has been reported to resist many antitumor agents but does not express P-gp (11). Two synthetic MDR1 siRNAs were transfected into COLO 320DM to determine if they could inhibit the expression of MDR1 mRNA and P-gp. Furthermore, cell proliferation analysis and intracellular adriamycin accumulation assays were performed after the introduction of MDR1 siRNAs combined with antitumor drug treatment in both cell lines.

\section{Materials and methods}

Cell culture. Two human colon adenocarcinoma cell lines, COLO 320DM and HT-29, were purchased from ATCC (American Type Culture Collection, Manassas, VA) and 
cultured in RPMI-1640 medium (Hyclone, Logan, UT) supplemented with 10\% FBS (Sigma, St. Louis, MO), 100 units $/ \mathrm{ml}$ penicillin and $100 \mu \mathrm{g} / \mathrm{ml}$ streptomycin (Mediatech Inc., Herndon, VA) at $37^{\circ} \mathrm{C}$ in a humidified incubator containing $5 \% \mathrm{CO}_{2}$.

siRNA synthesis and transfection. The siRNA sequences targeting ABCB1 (Genebank accession no. NM000927) were designed according to the algorithm developed by Ambion's partner Cenix BioScience GmbH (Dresden, Germany). Two MDR1 siRNA duplexes (\#4123 and \#4029 MDR1 siRNA), one positive control ( $\beta$-actin) siRNA, and one negative control siRNA were synthesized by Ambion (Austin, TX). For \#4123 MDR1 siRNA the sense strand is 5'-GGAUAUU AGGACCAUAAAUtt-3' and the antisense strand is 5'-AUU UAUGGUCCUAAUAUCCtg-3'. For \#4029 MDR1 siRNA the sense strand is 5'-GGAAAUUUAGAAGAUCUGAtt-3' and the antisense strand is 5'-UCAGAUCUUCUAAAUU UCCtg-3'. Cells treated with Lipofectamine ${ }^{\mathrm{TM}} 2000$ and Opti-MEM ${ }^{\circledR}$ I reduced serum medium (Invitrogen, Carlsbad, CA) were set as the liposome control (mock). The siRNA duplexes were transfected according to the recommended procedure by using the Lipofectamine 2000.

Real-time RT-PCR. Total RNA was isolated from cells using TRIzol Reagent (Invitrogen) according to manufacturer's instructions. Single stranded cDNA was synthesized from $1 \mu \mathrm{g}$ RNA using a cDNA synthesis kit (Invitrogen). The newly synthesized cDNA was amplified by PCR using an iCycle instrument (Bio-Rad, Hercules, CA). The reaction mixture contained $1 \mu \mathrm{l}$ of cDNA template, $1.5 \mathrm{mM} \mathrm{MgCl}_{2}, 2.5$ units Taq polymerase, 10 pmol MDR1 sense primer and 10 pmol MDR1 antisense primer, $0.63 \mu 1 \mathrm{SYBR}$ green I, and $0.25 \mu \mathrm{l}$ FCD (Roche, Florence, SC). The final reaction volume was $25 \mu 1$. Both sense and antisense $\beta$-actin primers were used as internal controls. Cycling conditions were as follows: $95^{\circ} \mathrm{C}$ for $5 \mathrm{~min}$ to denature cDNA and primers, then followed by 40 cycles at $95^{\circ} \mathrm{C}$ for $30 \mathrm{sec}, 55^{\circ} \mathrm{C}$ (MDR 1 ) or $56^{\circ} \mathrm{C}$ (ß-actin) for $30 \mathrm{sec}$ and $72^{\circ} \mathrm{C}$ for $30 \mathrm{sec}$. Based on the fluorescence intensity curve, the appropriate amplification cycles ( 26 cycles for MDR1 and 23 cycles for $\beta$-actin with the last cycle running at $72^{\circ} \mathrm{C}$ for $5 \mathrm{~min}$ ) were chosen. Aliquots of PCR product were electrophoresed using $1.5 \%$ agarose gels, and PCR fragments were visualized by ethidium bromide staining. The amplification products of MDR1 and B-actin were 161 and $254 \mathrm{bp}$, respectively.

Western blot analysis. Cells were washed twice with PBS, and then lysed with lysis buffer (30 mM Tris-HCl pH 7.4, $1 \%$ SDS). Cell lysates were centrifuged at $12000 \mathrm{~g}$, at $4^{\circ} \mathrm{C}$ for $15 \mathrm{~min}$. The total cellular protein was determined with $\mathrm{BCA}^{\mathrm{TM}}$ protein assay kit (Pierce, Rockford, IL). Subsequently, cellular proteins were dissolved in sample loading buffer and run on $7.5 \%$ SDS-PAGE gels. Proteins were electrotransferred onto PVDF membranes $(100 \mathrm{~V}, 2.5 \mathrm{~h})$. The membranes were rinsed with PBS and blocked with $10 \%$ non-fat milk in PBS for $3 \mathrm{~h}$ at room temperature. Membranes were then incubated with the primary antibody C219 (1:100) (Signet, El Monte, CA) or ß-actin (1:4000) (Sigma) in 3\% non-fat milk. After primary antibody incubation, membranes
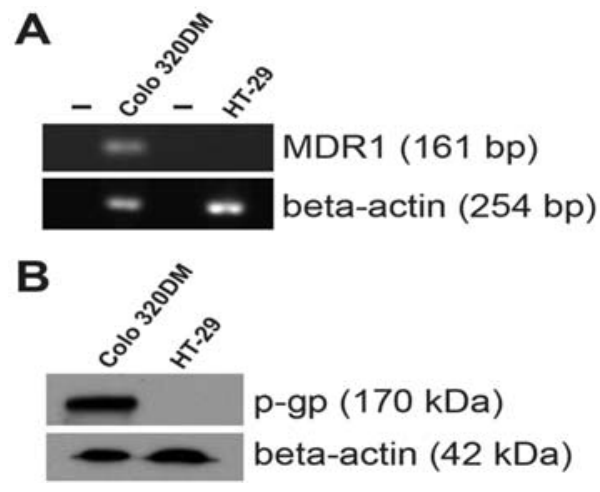

Figure 1. (A) RT-PCR analysis of MDR1 mRNA expression in COLO $320 \mathrm{DM}$ and HT-29 cell lines. MDR 1 mRNA was strongly detected in COLO 320DM, but not in the HT-29 control cell line. -, no cDNA template added. (B) Western blot analysis of P-gp with monoclonal antibody C219 in COLO 320DM and HT-29 cell lines. COLO 320DM expressed P-gp, but HT-29 did not.

were rinsed in TBS-T wash buffer for 5 min 6 times each. Membranes were then incubated with secondary antibody (HRP-conjugated goat anti-mouse IgG, 1:6000) (Pierce) for $1 \mathrm{~h}$ at room temperature and rinsed in TBS-T wash buffer for 5 min 6 times each. The protein-antibody complexes were visualized by chemiluminescence (ECL system, Amersham Bioscience, Piscataway, NJ).

MTT assay. Cell proliferation assays were performed by MTT assay (12). Cells were seeded at $1 \times 10^{4} /$ well in 96 -well microtiter plates. After $24 \mathrm{~h}$ incubation, siRNA transfection was performed. An antitumor drug was added $4 \mathrm{~h}$ after transfection. Then cells were incubated at $37^{\circ} \mathrm{C}$ in $5 \% \mathrm{CO}_{2}$. Then $30 \mu 1$ of $5 \mathrm{mg} / \mathrm{ml}$ MTT solution was added and incubated for $4 \mathrm{~h}$ at $37^{\circ} \mathrm{C}$. Medium and MTT solution were discarded after $4 \mathrm{~h}$ incubation. DMSO (150 $\mu \mathrm{l})$ was added into each well to stop the reaction and shaken for $5 \mathrm{~min}$. Optical density (OD) value was read on a Synergy HT multi-detection microplate reader (Bio-Tek Instruments, Inc., Winooski, VT) at $\lambda=570 \mathrm{~nm}$.

Intracellular adriamycin accumulation assay. The fluorescence intensity of intracellular adriamycin was determined using flow cytometry according to the standard method (13). Cells were plated at $2 \times 10^{5} /$ well in 6 -well plates $24 \mathrm{~h}$ prior to siRNA transient transfection and incubated for $48 \mathrm{~h}$. Then adriamycin $(10 \mu \mathrm{mol} / 1$ final concentration) was added and incubated for $2 \mathrm{~h}$. The cells were harvested and washed twice with cold PBS, then placed in ice-water to block the reaction until analysis. After half an hour, the fluorescence intensity of cells was determined by FACSCalibur ${ }^{\mathrm{TM}}$ flow cytometry (Becton-Dickinson, San Jose, CA) at an excitation wavelength of $488 \mathrm{~nm}$ and receiving wavelength of $575 \mathrm{~nm}$.

\section{Results}

Expression of MDR1 $m R N A$ and P-gp. In order to examine the MDR1 mRNA and P-gp expression in COLO 320DM and HT-29 cell lines, RT-PCR and Western blot analysis were performed. As shown in Fig. 1A, RT-PCR analysis revealed that COLO 320DM expressed MDR1 mRNA while HT-29 did not. The Western blot analysis was consistent with the results 


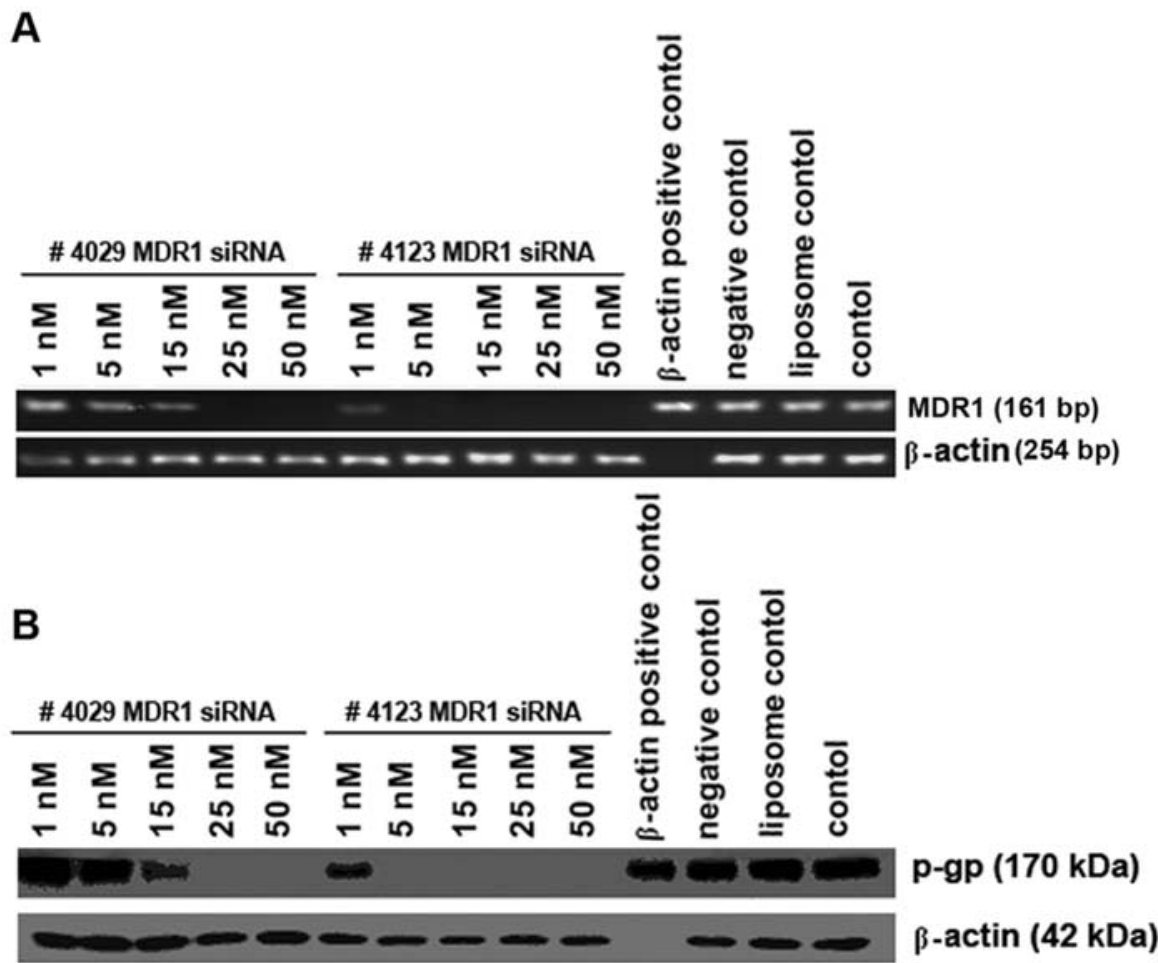

Figure 2. (A) Real-time RT-PCR analysis of MDR1 mRNA expression in COLO 320DM cell line with different concentrations of siRNA introduced into the cells. (B) Western blot analysis of P-gp in the COLO 320DM cell line. P-gp expression was not inhibited in the liposome, negative, or B-actin siRNA controls. But 50, 25, 15 and $5 \mathrm{nmol} / 1$ of \#4123 MDR1 siRNA completely inhibited the expression of P-gp. \#4029 MDR1 siRNA (50 and 25 nmol/l) completely inhibited the expression of P-gp as well.

from RT-PCR. Western blot analysis (Fig. 1B) also revealed that COLO 320DM had strong expression of P-gp while HT-29 had no detectable P-gp.

The minimum inhibition concentrations of MDRI siRNAs on MDR1 mRNA and P-gp expression in COLO 320DM cells. In order to investigate the inhibition effect of siRNA on the expression of MDR1 mRNA and P-gp, COLO 320DM cells were seeded in a 24-well plate. Serial concentrations of the two MDR1 siRNAs were transfected into COLO 320DM cells. Cells cultured in medium without liposome and siRNA treatment were used as controls. The liposome control (mock) was treated only with Lipofectame 2000. Positive controls were treated with $50 \mathrm{nmol} / \mathrm{l} \beta$-actin siRNA. Negative controls were treated with control siRNA containing no homology to any human gene. Cells of each group were harvested $72 \mathrm{~h}$ after transfection. Real-time RT-PCR detected expression of MDR1 mRNA. Western blot examined the expression of P-gp. Fig. 2A demonstrates the minimum concentration of siRNA required for complete inhibition of MDR1 mRNA expression. Cells were introduced to a $1-50 \mathrm{nmol} / \mathrm{l}$ range of siRNA concentrations. The minimum concentration for \#4029 MDR1 siRNA was $25 \mathrm{nM}$ while for \#4123 MDR1 siRNA it was $5 \mathrm{nM}$. The Western blot analysis of P-gp expression was consistent with the result from real-time RT-PCR (Fig. 2B).

The duration of inhibition effect of different concentrations of \#4123 MDR1 siRNA on P-gp expression in COLO 320DM cells. In order to investigate the onset time of MDR1 siRNA and the duration of inhibition after transient transfection with chemically synthesized MDR1 siRNA, \#4123 MDR1 siRNA at serial concentrations (higher than the minimum concentration) was transfected into COLO 320DM cells. Cells were harvested from 1 to 10 days after transfection. As shown by Western blot in Fig. 3, when COLO 320DM cells were transfected with $5 \mathrm{nM}$ of \#4123 MDR1 siRNA P-gp expression began to decrease after day 1 then increased from day 5 on. At $25 \mathrm{nM}$ of \#4123 MDR1 siRNA, P-gp expression was inhibited from day 1 to day 5; 25 and $50 \mathrm{nM}$ of \#4123 MDR1 siRNA had similar effects. At $100 \mathrm{nM}$ of \#4123 MDR1 siRNA, P-gp expression began to decrease after day 1 and then increased beginning at day 7 . Therefore, the effective treatment times of 5, 25 and $100 \mathrm{nmol} / \mathrm{l}$ of \#4123 MDR1 siRNA were 4,5 and 6 days, respectively.

Cell viability of COLO 320DM cells treated with different concentrations of \#4123 MDR1 siRNA and adriamycin at different time-points. Expressions of P-gp in COLO 320DM cells transfected with serial concentrations of \#4123 MDR1 siRNA were different. The higher the concentration of MDR1 siRNA, the longer the inhibition of P-gp expression. Adriamycin is one of the most common antitumor drugs. Of concern was whether the longer inhibition of P-gp expression when the cells were treated with adriamycin was consistent with COLO 320DM cell viability. Serial concentrations of \#4123 MDR1 siRNA were transfected into COLO 320DM cells, and $4 \mathrm{~h}$ after transfection, a final concentration of $0.32 \mu \mathrm{mol} / 1$ of adriamycin was added. Cells were cultured from 1 to 10 days. Cell viability was measured by MTT assay. As shown in Fig. 4, cell viability of COLO 320DM cells decreased to about $61 \%$ by day 10 when cells were treated with adriamycin only. But when cells were treated with 5, 25, 


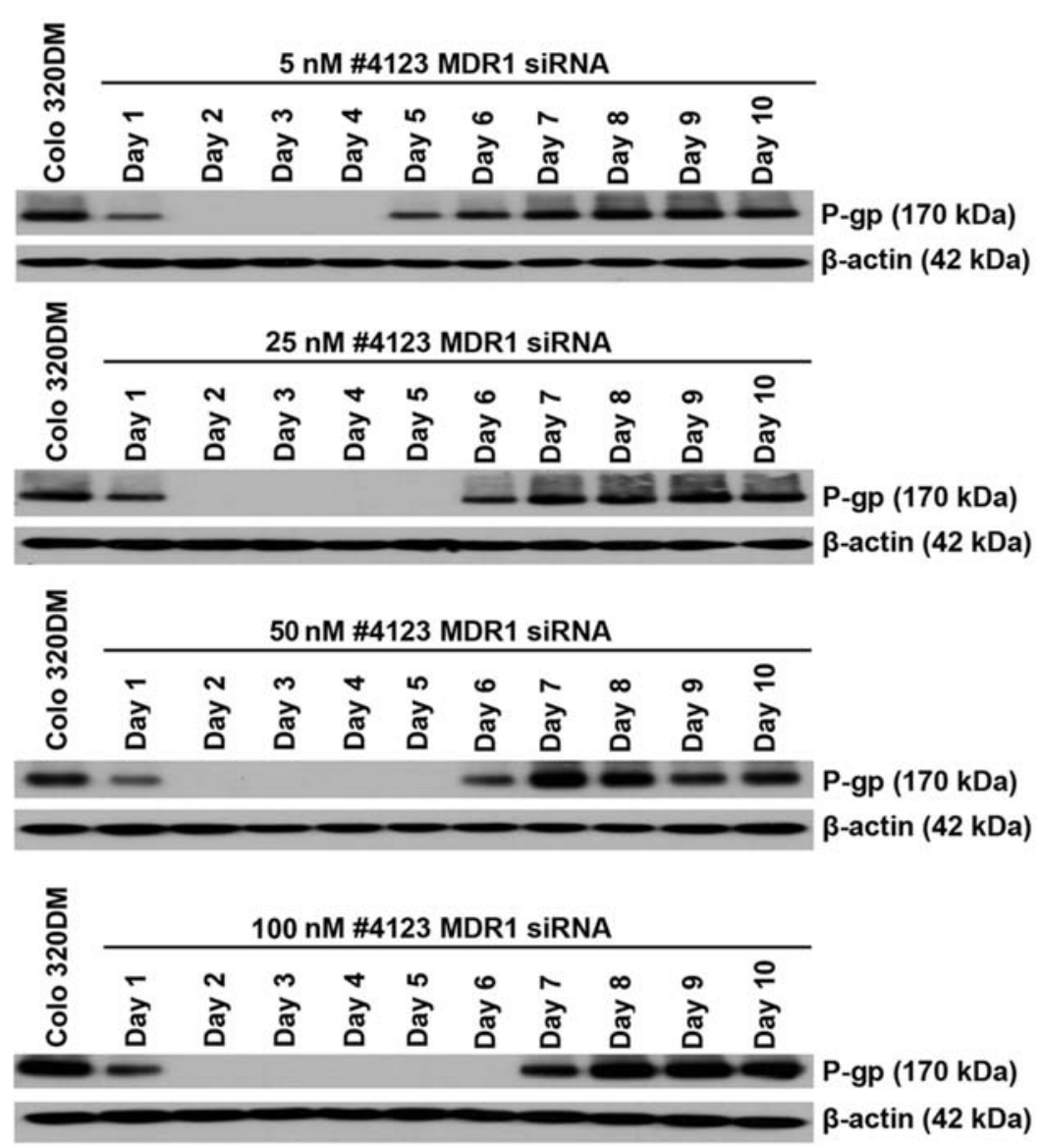

Figure 3. Western blot analysis of P-gp expression days 1 through 10 in COLO 320DM cells transfected with different concentrations of \#4123 MDR1 siRNA.

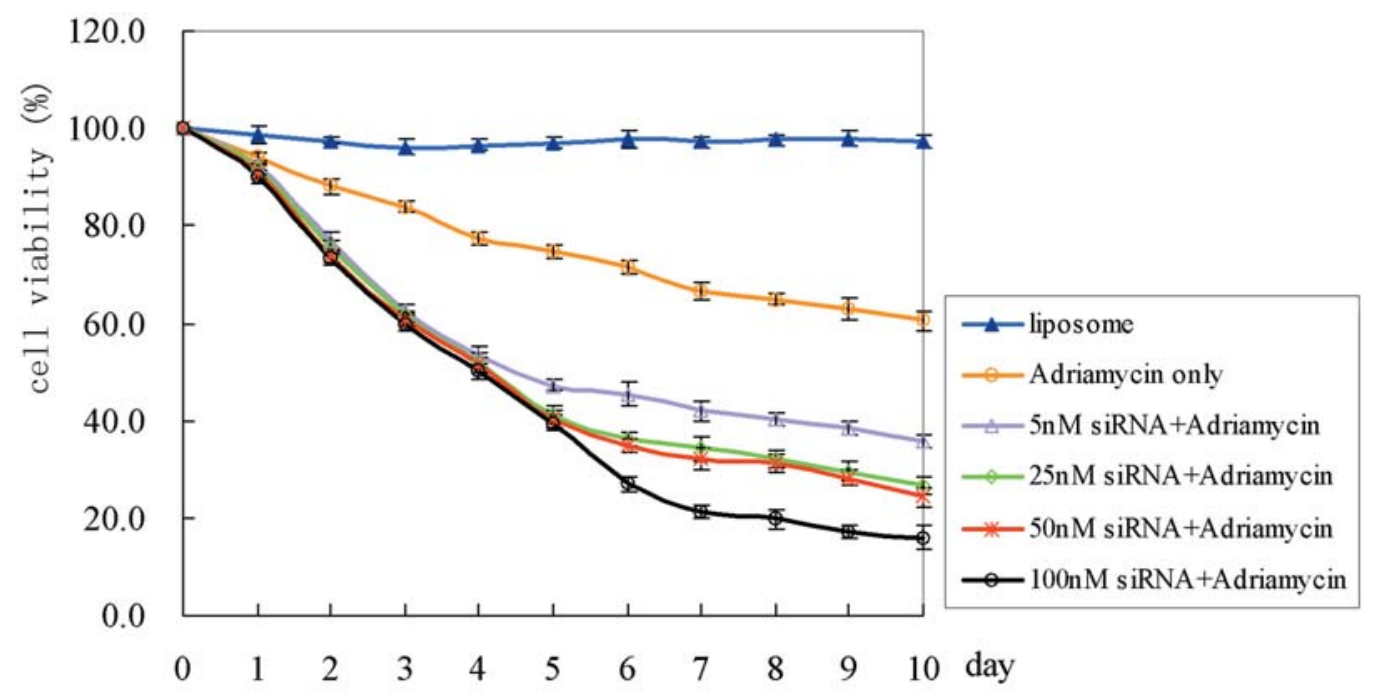

Figure 4. Cell viability of COLO 320DM cells treated with different concentrations of \#4123 MDR1 siRNA and adriamycin over a 10-day incubation.

50 and $100 \mathrm{nM}$ MDR1 siRNA combined with adriamycin, cell viability decreased to about $36,27,25$ and $17 \%$, respectively.

Enhancement of chemosensitivity of COLO 320DM cells treated with MDR1 siRNA. To investigate if MDR1 siRNA could reverse the MDR of the COLO 320DM cell line mediated by P-gp, COLO 320DM and HT-29 control cells were transfected with \#4123 or \#4029 MDR1 siRNA. Both cell lines were treated with the minimum concentrations $(5 \mathrm{nM}$ for \#4123 MDR1 siRNA, and 25 nM for \#4029 MDR1 siRNA) of MDR1 siRNAs, and then additionally treated with the antitumor agents adriamycin or vincristine respectively for 3 days. Adriamycin and vincristine were added to the cell culture in the following concentrations: 0.32 and 0.0128 $\mu \mathrm{mol} / 1$ respectively for the COLO 320DM cell line, 0.16 and $0.0016 \mu \mathrm{mol} / 1 \mathrm{respectively}$ for the HT-29 cell line. Finally 
Table I. Cell viability of COLO 320DM and HT-29 cells after MDR1 siRNA (\#4123 or \#4029) transfections combined with adriamycin and vincristine treatment.

COLO 320DM cell line (\%)

HT-29 cell line $(\%)$

\begin{tabular}{lcc}
\hline Control & $100.0 \pm 1.2$ & $100.0 \pm 4.3$ \\
Liposome only & $98.4 \pm 2.2$ & $99.8 \pm 4.0$ \\
\#4123 MDR1 siRNA & $97.8 \pm 3.1$ & $97.6 \pm 4.1$ \\
\#4029 MDR1 siRNA & $98.8 \pm 2.8$ & $96.8 \pm 9.5$ \\
Adriamycin & $83.2 \pm 2.7^{\mathrm{a}}$ & $80.1 \pm 5.0^{\mathrm{a}}$ \\
Liposome plus adriamycin & $81.9 \pm 3.7^{\mathrm{a}}$ & $80.4 \pm 4.7^{\mathrm{a}}$ \\
\#4123 MDR1 siRNA plus adriamycin & $63.4 \pm 1.7^{\mathrm{a}, \mathrm{b}}$ & $78.8 \pm 3.2^{\mathrm{a}}$ \\
\#4029 MDR1 siRNA plus adriamycin & $61.6 \pm 5.6^{\mathrm{a}, \mathrm{b}}$ & $78.9 \pm 4.8^{\mathrm{a}}$ \\
Vincristine & $84.3 \pm 6.5^{\mathrm{a}}$ & $82.7 \pm 7.1^{\mathrm{a}}$ \\
Liposome plus vincristine & $82.1 \pm 4.5^{\mathrm{a}}$ & $83.9 \pm 7.4^{\mathrm{a}}$ \\
\#4123 MDR1 siRNA plus vincristine & $59.8 \pm 7.1^{\mathrm{a}, \mathrm{c}}$ & $82.1 \pm 5.4^{\mathrm{a}}$ \\
\#4029 MDR1 siRNA plus vincristine & $62.6 \pm 9.9^{\mathrm{a}, \mathrm{c}}$ & $82.7 \pm 6.3^{\mathrm{a}}$ \\
\hline
\end{tabular}

${ }^{\mathrm{a} C}$ Compared with control, $\mathrm{P}<0.01$. ${ }^{\mathrm{b}} \mathrm{Compared}$ with adriamycin, $\mathrm{P}<0.01 .{ }^{\mathrm{c} C}$ Compared with vincristine, $\mathrm{P}<0.01$.

Table II. Fluorescence intensity of adriamycin in two cell lines after treatment of adriamycin combined with MDR1 siRNAs.

\begin{tabular}{lcc}
\hline & COLO 320DM cell line & HT-29 cell line \\
\hline Control & $4.25 \pm 0.37$ & $7.97 \pm 0.68$ \\
Adriamycin & $194.13 \pm 23.74$ & $247.74 \pm 18.23$ \\
Liposome plus adriamycin & $202.40 \pm 13.17^{\mathrm{b}}$ & $261.70 \pm 5.48^{\mathrm{b}}$ \\
\#4123 MDR1 siRNA plus adriamycin & $405.03 \pm 40.86^{\mathrm{a}}$ & $272.30 \pm 14.07^{\mathrm{b}}$ \\
\#4029 MDR1 siRNA plus adriamycin & $375.26 \pm 21.61^{\mathrm{a}}$ & $252.79 \pm 8.59^{\mathrm{b}}$ \\
\hline
\end{tabular}

${ }^{\mathrm{a} C}$ Compared with adriamycin, $\mathrm{P}<0.01 .{ }^{\mathrm{b}}$ Compared with adriamycin, $\mathrm{P}>0.01$.

the MTT assay was performed. Table I demonstrates that neither \#4123 nor \#4029 MDR1 siRNA itself could kill COLO 320DM or HT-29 cells. However, more COLO 320DM cells were killed when treated with either \#4123 or \#4029 MDR1 siRNA combined with adriamycin or vincristine than the HT-29 control cells were.

Increased intracellular adriamycin accumulation in COLO 320DM cells treated with MDR1 siRNAs. To investigate if MDR1 siRNA could increase intracellular antitumor drug accumulation, intracellular adriamycin accumulation assay was performed. As shown in Table II, in the COLO 320DM cell line, fluorescence intensity in cells without adriamycin treatment was only 4.25 (negative control, background). When treated with adriamycin, fluorescence intensity was 194.13. However, the fluorescence intensity was significantly increased to 405.03 and 375.26 when treated with adriamycin combined with \#4123 or \#4029 MDR1 siRNA respectively. In contrast, in the HT-29 control cell line fluorescence intensity without adriamycin treatment was 7.97 (negative control, background) and when treated with adriamycin, the fluore- scence intensity was 247.74 . However, the fluorescence intensity could not be significantly increased when treated with adriamycin combined with either \#4123 or \#4029 MDR1 siRNA.

\section{Discussion}

COLO 320DM is a multiple drug resistant (MDR) human colon cancer cell line which is resistant to many antitumor drugs including adriamycin and vincristine express P-gp, but not multidrug resistance-associated protein (MRP) (11). Therefore, COLO320DM is a MDR1/P-gp-dependent MDR colon cancer cell line. In contrast, HT-29 is a non-MDR1/ P-gp-dependent MDR human colon cancer cell line. HT-29 does not express P-gp (11), but exhibits MDR related to the expression of MRP (14). This study investigates the RNAi effects on the MDR of the COLO 320DM and HT-29 cell lines. There have been reports of RNAi knock down of MDR in colon cancer, namely, that RNAi decreased both MDR1 mRNA and P-gp. However, those studies were in different cancer cell lines, without a control cell line to demonstrate 
that RNAi worked directly through MDR1/P-gp $(15,16)$. In this study, we clearly demonstrate the efficacy of MDR1 siRNA targeting MDR1 mRNA in knocking down the phenotype of MDR in COLO 320DM cells, without knocking down MDR effects in the HT-29 control cells. Furthermore, MDR1 siRNAs enhance the cytotoxicity of the antitumor drugs adriamycin and vincristine in COLO 320DM but not in the HT-29 control cell line. This reveals the specificity of MDR1 siRNA targeting to MDR1 mRNA.

The MDR phenotype can result from the overexpression of members of the ATP binding cassette (ABC) superfamily of transmembrane transporters. The MDR phenotype is accompanied by enhanced activity of $\mathrm{P}-\mathrm{gp}$ and is designated classical MDR, while MDR phenotypes without P-gp are named atypical or non-MDR1/P-gp-dependent MDR (4). Several mechanisms lead to atypical MDR, including overexpression of alternative ABC-transporters, such as MRP, or breast cancer resistance protein (BCRP). In one report, the Caco- 2 colon cancer cell line was used as a model to investigate the degree of suppression siRNA targeting to MDR1 mRNA had on MDR1 gene expression and function in colon cancer cells. However, Caco-2 cells highly expressed BCRP and MRP2 in addition to P-gp as compared to classical MDR1 (15). We can speculate that various mechanisms were attributed to the MDR phenotype of the Caco- 2 cell line and that BCRP and MRP2 may play a more important role in leading to MDR in the Caco-2 cell line. Therefore, the Caco-2 cell line may not be the best colon cancer cell line to be used to investigate the suppressing effect of siRNA targeting to MDR1 mRNA on MDR1 gene expression and function. Based on our results, we strongly believe the COLO 320DM cell line is a better choice for investigating the effect of MDR1 siRNA on the MDR1 gene expression and function in colon cancer cells.

This study demonstrates that two synthetic MDR1 siRNAs (\#4123 and \#4029 MDR1 siRNA) can inhibit the expression of MDR1 mRNA and P-gp in the COLO 320DM cell line. These data suggest that silencing the expression of MDR1 mRNA and P-gp as mediated by MDR1 siRNA is specific and effective. The minimum concentration of \#4123 MDR1 siRNA which could completely inhibit the expression of MDR mRNA and P-gp is $5 \mathrm{nM}$; the minimum concentration of \#4029 MDR1 siRNA is $25 \mathrm{nM}$ (Fig. 2). The minimum concentrations of these two MDR 1 siRNAs are lower than the optimum concentrations of reported MDR1 siRNAs in both the studies of Peng et al and Wu et al $(17,18)$. This is likely due to the differences in the sequences of MDR 1 siRNAs and transfection. It is also well known that high concentrations of siRNA may not be efficacious because it could induce non-specific gene silencing. The effective concentration of siRNA is usually less than $100 \mathrm{nM}$ (19). Therefore, the minimum concentration of each siRNA in this study should be optimal.

The intracellular adriamycin accumulation assay demonstrated that both \#4123 and \#4029 MDR1 siRNA could increase intracellular adriamycin accumulation in COLO 320DM cells, but not in HT-29 cells. This is consistent with the MTT assay results. This indirectly indicates that these two MDR1 siRNAs can suppress the function of P-gp in MDR1/P-gp-dependent MDR colon cancer cells, but can not suppress the function of $\mathrm{P}$-gp in non-MDR1/P-gp-dependent MDR colon cancer cells.

However, the inhibiting effects of MDR1 siRNA on MDR1 mRNA and P-gp in the COLO 320DM cell line were obtained in vitro. Additionally, another clinical application issue should be considered: MDR1 gene products are present not only in malignant cells but also in some normal tissues such as peripheral blood cells and hemopoietic progenitors found in normal human bone marrow (20). Although MDR1 expression in these tissues is relatively low, P-gp expression may be important functionally in protecting these rapidly dividing cells from toxicity after exposure to anticancer drugs. Thus, in the clinical situation, it may be necessary that the application of the MDR1 siRNA expression vector be restricted to MDR1/P-gp-expressing multidrug-resistant cancer cells. One promising strategy for cancer cell-limited delivery of MDR1 siRNAs is the development of vector systems specific for multidrug-resistant cancer cells. Recently, a 'replication-defective' E1A-mutant adenoviral vector that selectively and efficiently replicates in 'classical' multidrugresistant cells has been described (21). Viruses like this may provide the basis for developing novel vectors for specific gene therapies for multidrug-resistant cancer cells.

\section{References}

1. Hawk ET, Limburg PJ and Viner JL: Epidemiology and prevention of colorectal cancer. Surg Clin North Am 82: 905-941, 2002.

2. Taa BG, van Tinteren $\mathrm{H}$ and Zoetmulder FA: Adjuvant 5FU plus levamisole in colonic or rectal cancer: improved survival in stage II and III. Br J Cancer 85: 1437-1443, 2001.

3. Redmond SM, Joncourt F, Buser K, et al: Assessment of Pglycoprotein, glutathione-based detoxifying enzymes and O6alkylguanine-DNA alkyltransferase as potential indicators of constitutive drug resistance in human colorectal tumors. Cancer Res 51: 2092-2097, 2001.

4. Germann UA, Pastan I and Gottesman MM: P-glycoproteins: mediators of multidrug resistance. Semin Cell Biol 4: 63-76, 1993.

5. Van de Vrie W, Gheuens EE, Durante NM, et al: In vitro and in vivo chemosensitizing effect of cyclosporin $\mathrm{A}$ on an intrinsic multidrug-resistant rat colon tumour. J Cancer Res Clin Oncol 119: 609-614, 1993.

6. Quattrone A, Papucci L, Morganti M, et al: Inhibition of MDR1 gene expression by antimessenger oligonucleotides lowers multiple drug resistance. Oncol Res 6: 311-320, 1994.

7. Kobayashi H, Dorai T, Holland JF, et al: Reversal of drug sensitivity in multidrug-resistant tumor cells by an MDR 1 (PGY1) ribozyme. Cancer Res 54: 1271-1275, 1994.

8. Damiani D, Michieli M, Michelutti A, et al: D-verapamil downmodulates P170-associated resistance to doxorubicin, daunorubicin and idarubicin. Anticancer Drugs 4: 173-180, 1993.

9. Lipardi C, Wei $\mathrm{Q}$ and Paterson BM: RNAi as random degradative PCR: siRNA primers convert mRNA into dsRNAs that are degraded to generate new siRNAs. Cell 107: 297-307, 2001.

10. Hannon GJ: RNA interference. Nature 418: 244-251, 2002.

11. Van Rensburg CE, Joone GK and O'Sullivan JF: Clofazimine and B4121 sensitize an intrinsically resistant human colon cancer cell line to P-glycoprotein substrates. Oncol Rep 7: 193-195, 2000.

12. Carmichael J, Degraff WG, Gazdar AF, et al: Evaluation of a tetrazolium-based semiautomated colorimetric assay: assessment of chemosensitivity testing. Cancer Res 47: 936-942, 1987.

13. Li J, Xu LZ, He KL, et al: Reversal effects of nomegestrol acetate on multidrug resistance in adriamycin-resistant MCF7 breast cancer cell line. Breast Cancer Res 3: 253-263, 2001.

14. Kok JW, Veldman RJ, Klappe K, et al: Differential expression of sphingolipids in MRP1 overexpressing HT29 cells. Int J Cancer 87: 172-178, 2000. 
15. Celius T, Garberg P and Lundgren B: Stable suppression of MDR 1 gene expression and function by RNAi in Caco- 2 cells. Biochem Biophys Res Commun 324: 365-371, 2004.

16. Pichler A, Zelcer N, Prior JL, et al: In vivo RNA interferencemediated ablation of MDR1 P-glycoprotein. Clin Cancer Res 11: 4487-4494, 2005.

17. Peng Z, Xiao Z, Wang Y, et al: Reversal of P-glycoproteinmediated multidrug resistance with small interference RNA (siRNA) in leukemia cells. Cancer Gene Ther 11: 707-712, 2004.

18. Wu H, Hait WN and Yang JM: Small interfering RNA-induced suppression of MDR1 (P-glycoprotein) restores sensitivity to multidrug-resistant cancer cells. Cancer Res 63: 1515-1519, 2003.
19. Semizarov D, Frost L, Sarthy A, et al: Specificity of short interfering RNA determined through gene expression signatures. Proc Natl Acad Sci USA 100: 6347-6352, 2003.

20. Klimecki WT, Futscher BW, Grogan TM, et al: P-glycoprotein expression and function in circulating blood cells from normal volunteers. Blood 83: 2451-2458, 1994.

21. Holm PS, Lage H, Bergmann S, et al: Multidrug-resistant cancer cells facilitate E1-independent adenoviral replication: impact for cancer gene therapy. Cancer Res 64: 322-328, 2004. 